\title{
Post-traumatic complications of arachnoid cysts and temporal lobe agenesis
}

\author{
T R K VARM A, C B SEDZIMIR, A N D J B MILES \\ From the Department of Medical and Surgical Neurology, Walton Hospital, Liverpool
}

\begin{abstract}
S U M M A R Y Six patients with arachnoidal cysts of the middle cranial fossa who presented with raised intracranial pressure are described. All of them had a history of trauma though in two of them it was a remote incident. They all showed radiological changes of an expanded middle cranial fossa and temporal lobe agenesis. The relationship of trauma to these congenital lesions is discussed.
\end{abstract}

Cysts overlying the temporal lobes have been well described in the literature. These are often associated with agenesis of the temporal lobe and their association with epilepsy and asymmetry of the skull is well known. We report our experience with six such cases, all of whom sustained minor trauma and suffered haemorrhagic complications.

Case 1

A 24 year old male nurse was admitted to a medical ward with a three day history of severe headaches and vomiting. He had marked nuchal rigidity with Kernig's sign. There were no lateralising signs or papilloedema. A provisional diagnosis of subarachnoid haemorrhage was confirmed when the CSF obtained at lumbar puncture was found to be xanthochromic. On later questioning he recalled a blow to the right temporal region sustained during a game of football a year previously. He had not lost consciousness or been otherwise disturbed.

Skull radiographs showed thinning and outward bulging of the right temporal bone with expansion of the middle cranial fossa.

Right carotid angiogram showed medial shift of the middle cerebral artery with appearances suggestive of a right temporal extracerebral collection.

Operation A right temporal burr hole was made and on incising the dura xanthochromic fluid gushed out under pressure. A right temporal craniotomy was then performed and a cystic cavity exposed. The superficial part of the cyst wall was adherent to the dura and the deep layer to arachnoid from which it was easily stripped. There appeared to be a direct communication between the cyst and the chiasmatic

Address for reprint requests: Mr TRK Varma, Department of Neurosurgery, Royal Hallamshire Hospital, Glossop Road, Sheffield S10 2JF. Accepted 30 July 1980 cistern. The temporal lobe was hypoplastic and the internal carotid artery, the optic nerve and the third nerve were easily visible without retraction. The patient recovered completely and ten years later was symptom free.

Case 2

A 7 year old boy was admitted with a six weeks history of headaches and vomiting. Two days prior to the onset of symptoms he had fallen from a fence and bumped his head. He had not lost consciousness and was not taken to hospital. There was no significant past illness and his development had been normal. He was fully conscious but had bilateral papilloedema though no lateralising signs.

Skull radiographs showed expansion of the right middle cranial fossa with outward bulging of the squamous temporal bone.

Bilateral carotid angiogram showed the midline vessels to be shifted to the left with evidence of an extracerebral collection in the right front temporal region.

Operation A right temporal burr hole was made and on opening the dura xanthochromic fluid gushed out. The child improved after this procedure but there was a persistent collection of fluid under the scalp wound which required repeated aspiration. Four months later a temporal craniotomy was performed. A cystic cavity was present replacing the tip of the agenetic temporal lobe. The walls of the cyst were easily stripped from the dura superficially and arachnoid on its inner aspect. The cyst appeared to communicate with general subarachnoid space by a "valvular" arrangement and by stripping its walls a good communication was established with the chiasmatic cistern. The child recovered well from the operation apart from a wound infection which was treated with anti-biotics. He was well two years later. 
Case 3

A 7 year old male child was admitted with a history of headache and vomiting for five weeks. He was the first born of twins and had no birth trauma. His development and intellect were normal. A few days after hospitalisation the mother recalled that the child had been knocked down by a slow moving taxi about twelve months prior to the onset of his symptoms. He had not lost consciousness and was not taken to hospital. On admission he was drowsy with a mild hemiparesis and early papilloedema.

Skull radiographs were at first thought to be normal but review showed there was thinning and outward bulging of the left squamous temporal bone with expansion of the middle cranial fossa.

Isotope brain scan was normal.

Carotid angiography showed a large extracerebral collection on the left side with elevation of the middle cerebral artery and shift of midline vessels.

Operation Three burr holes were made and on opening the dura $75 \mathrm{ml}$ of golden yellow fluid was drained. The cortical surface was about $1.5 \mathrm{~cm}$ from the inner table. There was a rapid improvement in his level of consciousness and relief from headache. However, there was a persistent collection of fluid over the burr holes. A week later a craniotomy was carried out and a large cyst found in the middle fossa replacing the tip of the temporal lobe. The cyst appeared to com- municate with the subarachnoid space through a valvular arrangement. The walls of the cyst were stripped exposing a large Sylvian fissure with a clear view of the optic chiasma and internal carotid artery. The patient had an uneventful recovery and remains well one year later.

\section{Case 5}

A 17 year old male was admitted complaining of frontal headache for two weeks. Five weeks prior to the onset of headache, after a game of rugby football, he had noticed a large bruise over the left supraorbital region. He attributed this to bumping his head in a tackle but he had not lost consciousness and did not go to hospital. He was found to have bilateral papilloedema and a mild right hemiparesis.

Skull radiographs showed expansion of the right middle fossa with outward bulging of the squamous temporal bone.

Carotid angiogram showed evidence of a right sided extracerebral collection.

Operation A right temporal craniotomy was carried out. A lightly bloodstained subdural collection of fluid was found with a $2 \mathrm{~cm}$ defect connecting it through a layer of apparently thickened arachnoid to a cavity in the anterior middle fossa. The anterior pole of the right temporal lobe was deficient but the exposed gyri appeared normal. The cyst was drained

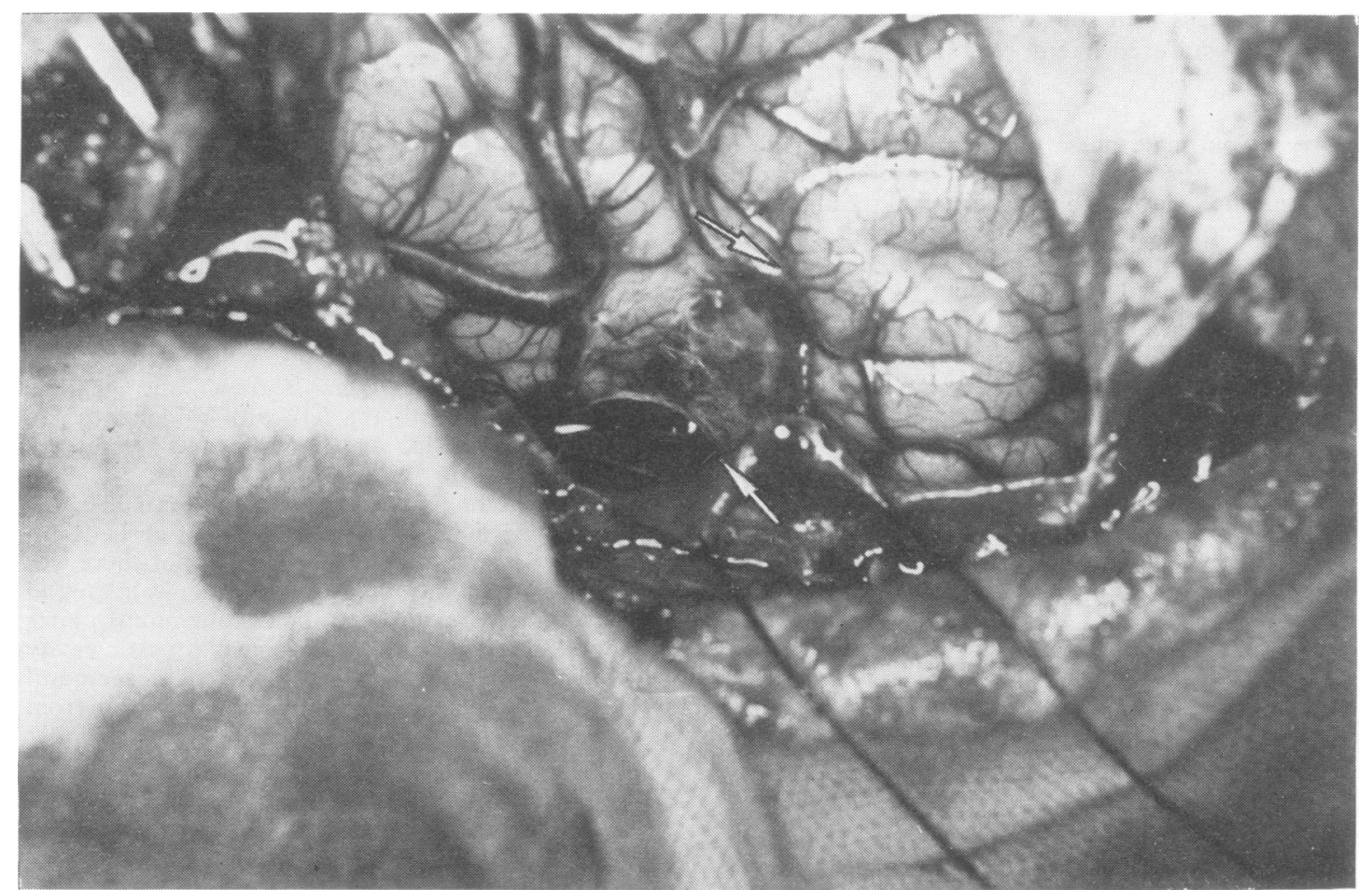

Fig 1 Operative photograph showing cyst wall with a tear in it. The temporal lobe is deficient in its anterior part leaving the sylvian fissure widely open. 


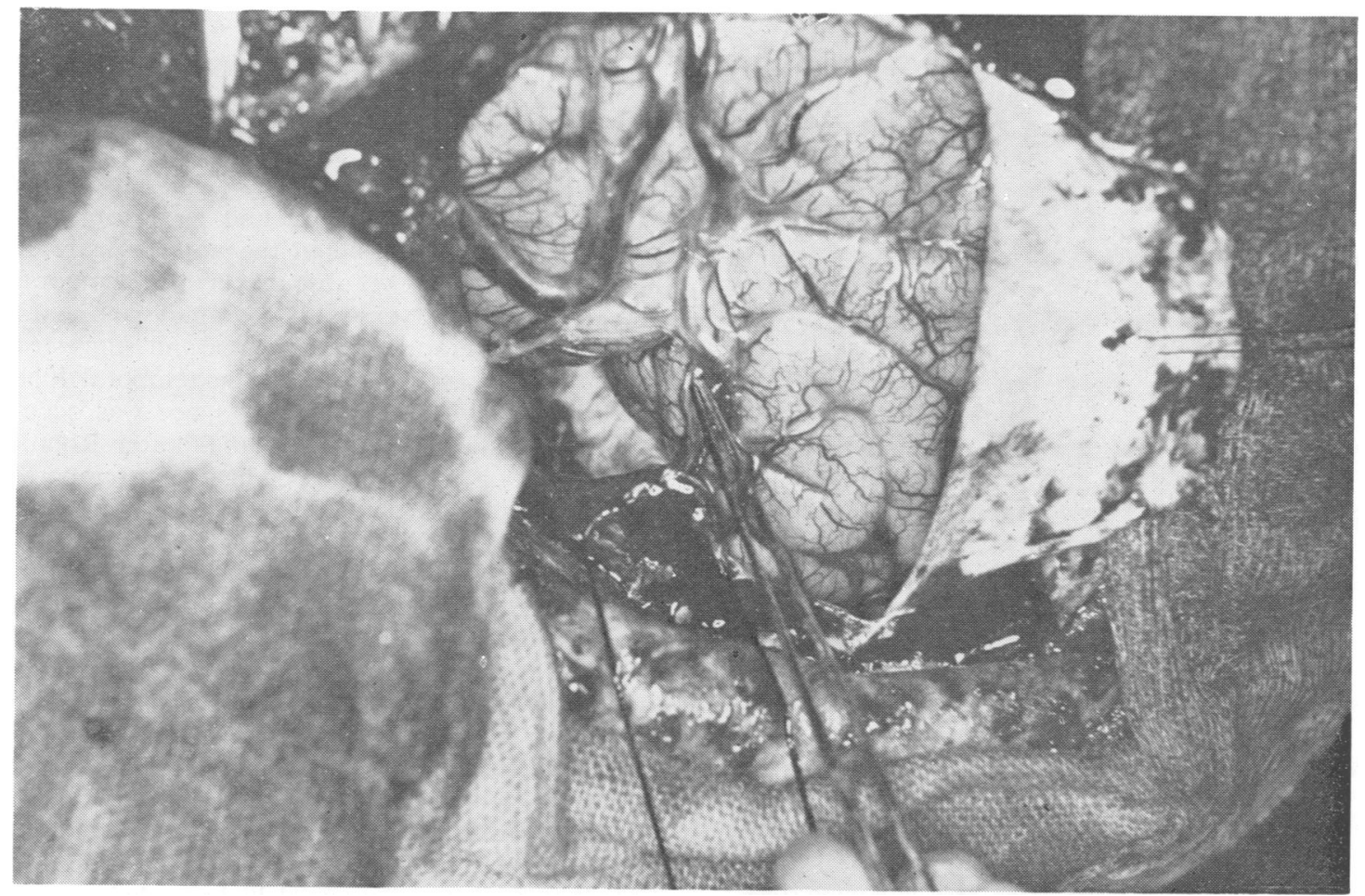

Fig 2 Same case as fig 1 after stripping off the cyst wall.

and the walls excised (figs 1 and 2). The patient had an uneventful recovery and was symptom free two years later.

\section{Case 5}

A 21 year old male, who was initially seen as an outpatient with a history of severe headache for one week. Four months prior to this he had fallen from his motorcycle but did not think he had banged his head. He was seen in hospital and treated for bruises of his back. At this stage he had no neurological deficit except for an equivocal plantar reaction on the left side. When seen again two weeks later the headaches were worse and he now had bilateral papilloedema with left hemiparesis.

Skull radiographs showed asymmetrical bulging of the right middle cranial fossa with thinning of the temporal bone.

Isotope brain scan was normal.

Right carotid angiography showed a large extracerebral collection on the right side.

Operation A large subdural haematoma containing black fluid was found overlying the parietal region. In the temporal region there was a tense cyst containing clear fluid and the tip of the temporal lobe was deficient. There was no connection between the two cavities though the haematoma did partially overlie the posterior edge of the cyst. The arachnoidal cyst did not appear to communicate with the general subarachnoid space. The walls of the cyst were stripped easily from the arachnoid and dura. The patient had an uneventful recovery and was well one year later.

\section{Case 6}

A 13 year old boy was admitted with a two month history of headache, which in the last two weeks had become severe and was associated with vomiting. Prior to the onset of symptoms the child had fallen from a skateboard but had not lost consciousness. He was conscious with hyper-reflexia and impaired sensation in the left limbs. He had florid bilateral papilloedema. Skull radiographs showed expansion of the right middle fossa with outward bulging of the right temporal bone.

$C A T$ scan showed small lateral ventricles displaced from the right by a large CSF density lesion in the frontal and temporal region associated with a bulge of the skull (figs 3 and 4).

Operation Right fronto-temporal craniotomy was performed. All the bones here were thin suggesting a long standing pressure on this part of the skull. Underlying the dura was an enormous cystic collection of haemorrhagic fluid contained in two cavities. The capsule of the cyst was adherent to the dura on the lateral aspect and to the arachnoid on the deep aspect, with a similar membrane partitioning the two cavities. 

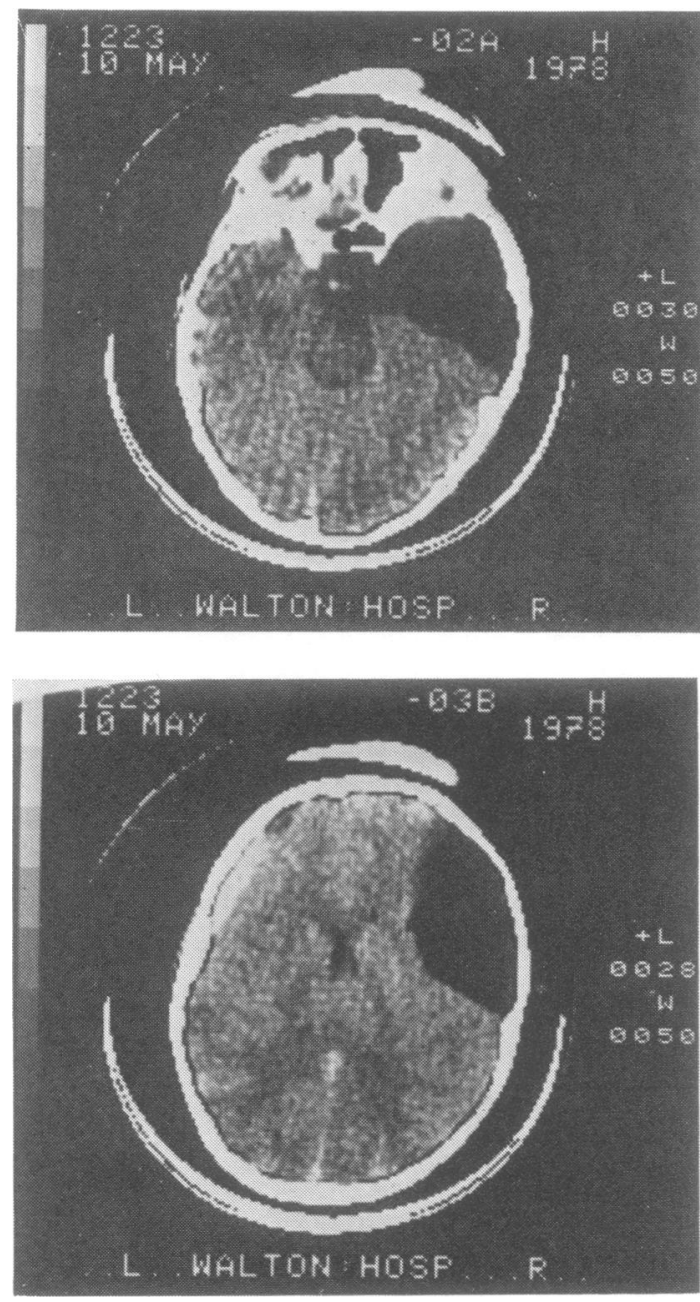

Fig 3, 4 CAT scan showing expansion of right middle cranial fossa with a low density arachnoidal cyst

The tip of the right temporal lobe was deficient. The walls of the cyst were excised to produce a free communication between the "cyst" and the general cerebro spinal fluid circulation. The patient recovered satisfactorily and was well two years later.

\section{Discussion}

Cysts overlying the cerebral hemispheres have been well recorded in literature and various aetiological factors suggested including trauma, infection and a congenital maldevelopment. Our six cases show four common features-their situation in the middle cranial fossa, the association with agenesis of the temporal lobe, asymmetry of the skull and a history of trauma.

Reynolds, ${ }^{12}$ Naffziger $^{3}$ and Frazier ${ }^{4}$ have recorded cases of patients with collections of fluid in the temporal region. Robinson ${ }^{5}$ reported two cases who presented with localised bulging of the skull in the temporal region and were found to have a collection of fluid in the anterior part of the middle fossa. The absence of the temporal lobe left the insula exposed. He analysed the 15 cases in the literature at that time and suggested that in these cases the tip of the temporal lobe was congenitally absent and the space was occupied by a cystic collection of fluid. Later Robinson $^{6}$ suggested that these cases had the "temporal lobe agenesis" syndrome and he discussed in detail the postmortem findings in one such case (case 10).

The exact relationship of the cyst to the meninges and their communication with the general subarachnoid space has been a matter of debate. Starkman et al, ${ }^{7}$ reporting the gross pathology and histopathology of four cases of cysts overlying the cerebral hemispheres, concluded that they are situated between two layers of arachnoid and separated from the pia mater by the subarachnoid space. However, Robinson, ${ }^{56}$ in his papers maintains that they are subarachnoidal in position and regards it as a true "external hydrocephalus." At operation the "cyst" appears to lie between the dura and the arachnoid. The walls, which appear macroscopically similar to the membrane around a chronic subdural haematoma, microscopically consist of a "vascular membrane." But this sheds no light on the origin of the membrane or its location.

Trauma as a precipitating factor has been a common finding in many cases in the literature. Robinson $^{5}$ in his review of cases in the literature at that time found a history of trauma in seven out of the 15 cases. Tiberin and Gruzkiewicz ${ }^{8}$ described a 10 year old boy with an arachnoidal cyst who had sustained a minor though significant head injury when he was two years old. Grüss et $a l^{9}$ reported a case of a 5 year old child with an arachnoidal cyst and temporal lobe agenesis. The child had sustained a severe head injury when he was one and a half years old but they felt this had no relevance to the development of the cyst. Four cases described by Törma and Heiskanen ${ }^{10}$ had a history of head injuries, one four years and the other four months prior to clinical presentation.

In our series all six cases had a history of head injury with varying time intervals prior to presentation. Two (cases I and II), like those quoted in 
the literature, had sustained the injury a year or more prior to the onset of symptoms. In each of these the head injury had been slight and not requiring medical attention. At operation fluid had been xanthocromic indicating that haemorrhage had been a factor in the presentation but whether this was traumatic or a spontaneous event seems a matter for speculation. The other four cases suffered a definite head injury more recently and these incidents would seem very likely to be of significance. The trauma was of a minor nature and only one was seen in hospital as a direct result of the injury.

Asymmetry of the skull as seen on skull radiographs was a characteristic feature of all our cases. In the earlier cases it was identified only in retrospect but later was recognised early and alerted us to the possibility of a temporal lobe agenesis and the possibility of a subdural collection. The radiological features that were noticed were the outward bulging of the squamous temporal bone and greater wing of the sphenoid which was seen best on the axial view (fig 5). The lateral view shows an anterior expansion of the middle cranial fossa by a forward displacement of the lesser and greater wings of the sphenoid (fig 6). Hardman ${ }^{11}$ discussed asymmetry of the

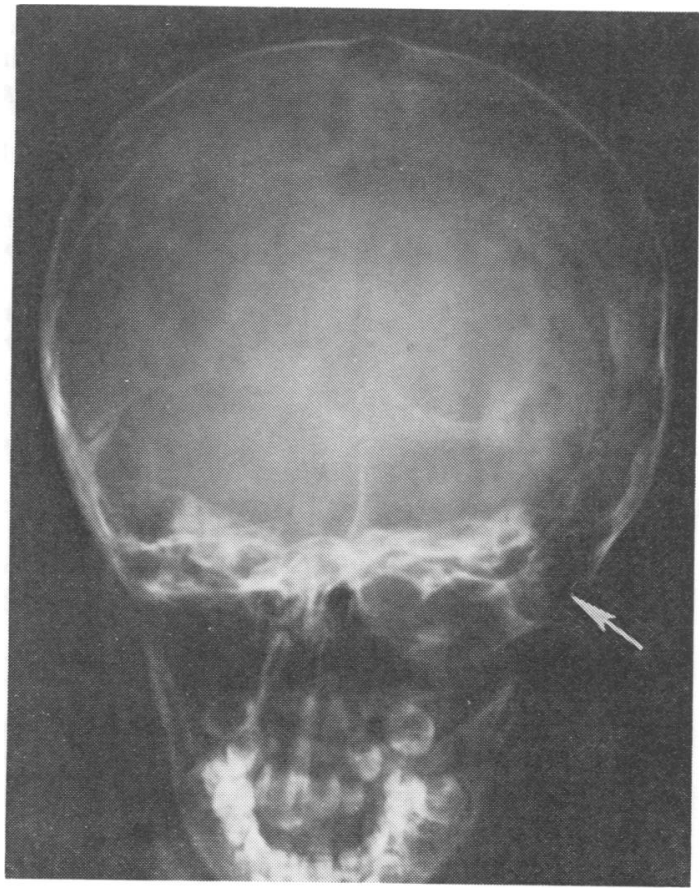

Fig 5 Axial skull x-ray showing bulging of left temporal bone skull in relation to subdural collection of fluid and described three cases, two of which were subdural haematomas and the other a "subdural hydroma." All three presented after trauma but one had noticed the swelling seven years prior to the injury. Childe"12 reported eight cases of "subdural hydromas," with localised thinning and bulging of the middle fossa and in these cases the swelling was the presenting symptom. In 10 of the 15 cases reviewed by Robinson ${ }^{5}$ the patients had themselves noticed the bulging of the skull; in eight of them it was the only clinical finding. The two cases he reported in 1955 presented with a temporal swelling, in one it was noticed after trauma and in the other appeared spontaneously. In his latest paper ${ }^{6}$ he considered the thinning and bulging of the temporal bone to be a diagnostic feature of the "temporal lobe agenesis" syndrome. Anderson and Landing ${ }^{13}$ discussed cerebral arachnoidal cysts in nine patients, eight of whom were children. All showed radiological thinning and expansion of the temporal bone but it was clinically distinguishable in only four of them. He suggested that such cysts were a common cause of rapid enlargements of the head in infants.

The actual cause of the expansion of the skull overlying these cysts is uncertain. Dr List, ${ }^{12}$ in his discussion on Childe's paper, suggested that it was due to the pulsating pressure of the localised collection of fluid. Robinson ${ }^{5}{ }^{6}$ suggests that the maldevelopment of the temporal lobe leaves a capacious subarachnoid space and suggests that

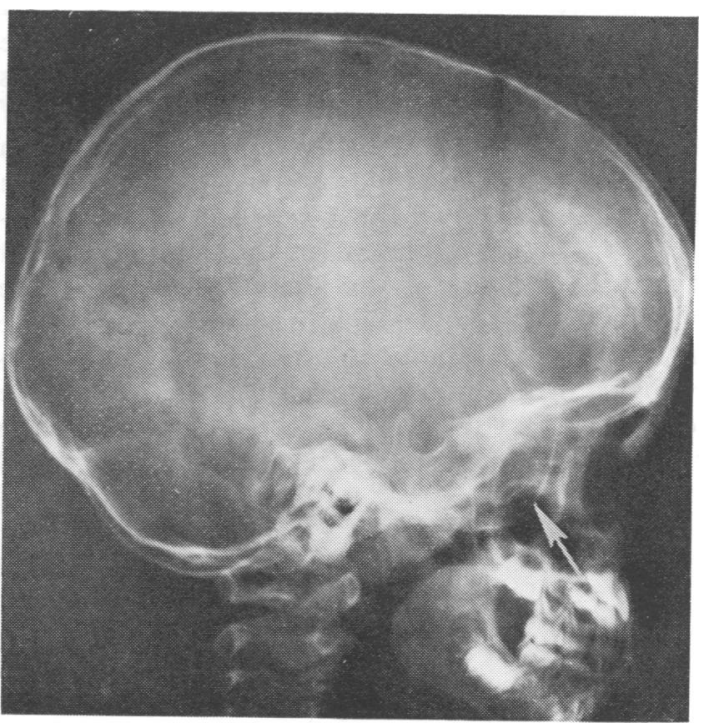

Fig 6 Lateral skull $x$-ray showing anterior expansion of middle cranial fossa 
the skull is expanded by its pulsation. This would have to occur before the skull is fully developed and suggests that the basic abnormality is more likely to be congenital.

Various clinical presentations of arachnoidal cysts of the middle fossa have been described, the commonest being epilepsy. All our cases except one presented with symptoms and signs of raised intracranial pressure and space occupation. Case 1 presented with subarachnoid haemorrhage. Torma and Heiskanen ${ }^{10}$ described a similar case in a 47 year old female, who presented as a verified subarachnoid haemorrhage with the history of a concussion suffered 11 months earlier. Robinson had a similar case in his series. These cysts may remain asymptomatic and be found at necropsy following death due to other causes. ${ }^{56}$

\section{Conclusions}

The six cases in our opinion, are examples of congenital agenesis of the temporal lobe and its susceptibility to haemorrhagic complications even with minor trauma.

It is likely that in most or all the cases minor trauma in some way provoked an increase in the size of the cyst. It is likely that trauma can give rise to haemorrhage within the cyst, causing this to expand. The source of the bleeding may be the vessels in the arachnoid or the cyst capsule. The cyst wall might also tear giving rise to a subdural collection of haemorrhagic fluid as was seen unequivocally in case 5 . It is also possible that tear of the deeper layer of the cyst wall gives rise to a valvular arrangement whereby CSF flows one way causing a gradual increase in the size of the cyst. It appears that these patients have an increased susceptibility to trivial trauma, an opinion also expressed in Greenfield's Neuropathology. ${ }^{14}$ The exact reason for this is not clear but it may be because of the altered anatomy of the middle cranial fossa. This may be similar to the increased vulnerability to trauma seen in hydrocephalus or in cerebral atrophy. There is evidence to suggest that the basic defect, that is either the cyst or the temporal lobe agenesis, is congential in origin. The expanded middle fossa certainly suggests long standing or life long abnormality. These changes can be seen on the plain radiographs. The haemorrhagic complication of a long standing abnormality may thus be recognised at an early stage.

Craniotomy and excision of the cyst walls would appear to be the ideal treatment. Drainage of the cyst through burr holes may be followed by recurrent accumulation of fluid as was seen in two of our cases.

\section{References}

1 Reynolds CE. Infective external hydrocephalus: shifting epilepsy. Br Med J 1921; 2:66-70.

2 Revnolds CE. Further observation upon external hydrocephalus. Br Med J 1922; 1:950.

3 Naffziger HC. Subdural fluid accumulations following head injury. JAMA 1924; 82:1751-2.

4 Frazier $\mathrm{CH}$. Cerebral pseudotumours. Arch Neurol 1930; 24:1117-32.

5 Robinson RG. Intracranial collections of fluid with local bulging of the skull. J Neurosurg 1955; 12:345-53.

6 Robinson RG. The temporal lobe agenesis syndrome. Brain 1964; 88:87-106.

7 Starkman SP, Brown TC, Lunell MD. Cerebral arachnoid cysts. J Neuropathol Exp Neurol 1958; 17:484-500.

8 Tiberin P, Gruzkewicz J. Chronic arachnoidal cysts of the middle cranial possa and their relation to trauma. J Neurol Neurosurg Psychiatry 1961; 24:86-91.

9 Grüss P, Auer-Doinet G. Genesis of temporal arachnoid cysts. Neuropaediatrie $1974 ; 5: 175-80$.

10 Torma T, Heiskanen O. Chronic subarachnoidal cyst in the middle cranial fossa. Acta Neurol Scand 1962; 38:166-70.

11 Hardman J. Asymmetry of the skull in relation to subdural collections of fluid. B J Radiol 1939; 12:455-61.

12 Childe AE. Localised thinning and enlargement of the cranium with special reference to the middle fossa. Am J Roentgenol 1953; 70:1-22.

13 Anderson FM, Landing BH. Cerebral arachnoid cysts in infants. J Paediatr 1975; 68:88-96.

14 Urich H. In: Backwood W \& Corsellis JAN (ed) Greenfield's Neuropathology, London: Edward Arnold 1976; 394. 